Pacific Journal of Mathematics

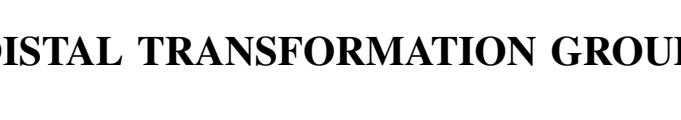




\title{
DISTAL TRANSFORMATION GROUPS
}

\author{
ROBERT ELLIS
}

Let $X$ be a topological space and $G$ a group of homeomorphisms of $X$ onto itself. Then $G$ is said to be distal if given any three points $x, y, z$ in $X$ and any filter $\mathscr{F}$ on $G$, then $x \mathscr{F} \rightarrow z$ and $y \mathscr{F} \rightarrow z$ implies that $x=y$. The above definition of distal is a topological variant of the one given in [2]; the two notions coincide when the underlying space $X$ is compact.

This paper deals with two topics in the study of distal transformation groups. First, a recursive characterization of these groups is given in a general setting, and second it is shown that under suitable restrictions on $X$ and $G$, distal is a property strong enough to imply equicontinuity of $G$. In order to make this statement precise a few definitions are needed. For a complete discussion of the following notions, the reader is referred to [2].

Let $a, b$ be functions of $X$ into $X$ and let $x \in X$. Then $x a$ will denote the image of $x$ under $a$, and $a b$ the composite function first $a$ then $b$. Under the operation of composition $X^{x}$ is a semigroup such that the maps $b \rightarrow a b\left(b \in X^{X}\right)$ are continuous for all $a \in X^{X}$, and the maps $b \rightarrow b a\left(b \in X^{X}\right)$ are continuous for all continuous functions $a$ of $X$ into $X$. The group $G$ may be regarded as a subset of $X^{X}$ and its closure $T$ formed. One may also consider $S$ the closure of $G$ in the topology of uniform convergence on $X$. When $X$ is compact, $S$ is a topological group of homeomorphisms of $X$ onto $X$ but is in general not compact, whereas $T$ is compact but is in general not a group. Hence in studying $T$ instead of $S$ the emphasis is on the algebraic rather than the topological structure.

A subset $A$ of $G$ is said to be syndetic if there exists a compact subset $K$ of $G$ such that $A K=G$. (If no topology is specified for $G$, then it is assumed to be provided with the discrete topology.) A point $x \in X$ is an almost periodic point with respect to $G$ if given any neighborhood $U$ of $x$, there exists a syndetic subset $A$ of $G$ such that $x A=[x a \mid a \in A] \subset U$. If every point of $X$ is an almost periodic point with respect to $G$, then $G$ is said to be pointwise almost periodic.

Let $I$ be a set with cardinal number $a>0$. Then each $g \in G$ induces a homeomorphism $\left(x_{i} \mid i \in I\right) \rightarrow\left(x_{i} g \mid i \in I\right)$ of $X^{\mathfrak{a}}$ onto $X^{\mathfrak{a}}$ which will also be referred to as $g$. Under this identification $G$ becomes a group of

Received March 14, 1958. This research was supported by the United States Air Force through the Air Force Office of Scientific Research of the Air Research and Development Command, under contract No. $A F 18$ (600)-1116. Reproduction in whole or in part is permitted for any purpose of the United States Governmennt. 
homeomorphisms of $X^{\mathfrak{a}}$ onto $X^{\mathfrak{a}}$.

The characterization mentioned in the second paragraph is that if $x G$ is compact for all $x \in X$ and $X$ is Hausdorff, then $G$ is distal if and only if $G$ is pointwise almost periodic on $X^{\mathfrak{a}}$ for all cardinals $\mathfrak{a}>0$.

The following lemma is probably well-known but the proof is included for the sake of completeness. For references to the literature see [3].

LEMMA 1. Let $S$ be a compact Hausdorff space with a semigroup structure such that the maps $s \rightarrow t s(s \in S)$ are continuous for all $t \in S$. Then there exists an idempotent $u \in S$.

Proof. Let $\mathscr{E}$ denote the class of non-null compact subsets $E$ of $S$ such that $E^{2} \subset E$. Then $\mathscr{E} \neq \phi$ since $S \in \mathscr{E}$. If $\mathscr{E}$ is ordered by inclusion, an application of Zorn's lemma shows that there is a minimal element $A$ in $\mathscr{E}$. If $r \in A$, then $r A$ is a non-null compact subset of $S$ such that $r A \in \mathscr{E}$ and $r A \subset A$. Hence $r A=A$ since $A$ is minimal. Thus there exists $p \in A$ with $r p=r$. Define $L=[a \mid a \in A$ and $r a=r]$. Then $p \in L$, and $L$ is a compact subset of $A$. Moreover $k$, $1 \in L$ imply that $r k 1=r 1=r$; that is $L^{2} \subset L$. Thus $L \in \mathscr{E}$ and so $L=A$. Hence $r \in L$; that is $r^{2}=r$. The proof is completed.

THEOREM 1. Let $X$ be a Hausdorff space and $G$ a group of homeomorphisms of $X$ onto $X$ such that $\overline{x G}$ is compact for all $x \in X$. Then the following statements are pairwise equivalent.

(1) The closure $T$ of $G$ in $X^{X}$ is a compact group.

(2) For every cardinal $\mathfrak{a}>0, G$ is pointwise almost periodic on $X^{\mathfrak{a}}$.

(3) There exists a cardinal $a>1$ such that $G$ is pointwise almost periodic on $X^{\mathfrak{a}}$.

(4) The group $G$ is distal.

Proof. (1) implies (2). Let $a$ be a cardinal $>0$, and let $I$ be a set of cardinal $a$. Let $x=\left(x_{i} \mid i \in I\right) \in X^{I}$ and $U$ a neighborhood of $x$. Then there exists a finite subset $J$ of $I$ and open subsets $V_{i}(i \in J)$ of $X$ such that $x \in W=\times\left(W_{i} \mid i \in I\right) \subset U$ where $W_{i}=V_{i}(i \in J)$ and $W_{i}=X(i \in I-J)$. Let $N=\left[t \mid t \in T\right.$ and $\left.x_{i} t \in V_{i}(i \in J)\right]$. Then $N$ is an open neighborhood of the identity $e$ of $T$. Let $t \in T$. Since the map $r \rightarrow r s(r \in T)$ of $T$ onto $T$ is a homeomorphism for all $s \in T, t^{-1} N$ is a non-null open subset of $T$. Hence there exists $g \in G$ such that $g \in t^{-1} N$; that is $t \in N g^{-1} \subset N G$. Thus $T \subset N G$, and so $T \subset N K$ for some finite subset $K$ of $G$. Since $G$ is a subgroup of $T$ and $K \subset G, G \subset(N \cap G) K$. Thus $A=N \cap G$ is a syndetic subset of $G$ with $x A \subset U$.

That (2) implies (3) is clear.

(3) implies (4). Let $x, y, z \in X$ and let $\mathscr{F}$ be a filter on $G$ such 
that $x \mathscr{F} \rightarrow z$ and $y \mathscr{F} \rightarrow z$. Let $a$ be a cardinal $>1, I$ a set of cardinal a, and $i$ and $j$ two distinct elements of $I$. Let $w=\left(w_{k} \mid k \in I\right) \in X^{\mathfrak{a}}$ such that $w_{k}=x, k \neq j$ and $w_{j}=y$. Then $w \mathscr{F} \rightarrow u=\left(u_{k} \mid k \in I\right)$ where $u_{k}=z(k \in I)$. Hence $u \in w G \subset \times\left(w_{k} G \mid k \in I\right)$. Thus $u \in \bar{w} G$ which is a compact set on which $G$ is pointwise almost periodic. Therefore by $[2 ; 4.07] w \in \bar{u} G$. Consequently there exists a filter $\mathscr{G}$ on $G$ such that $u \mathscr{G} \rightarrow w$; that is $z \mathscr{G} \rightarrow x$ and $z \mathscr{G} \rightarrow y$. Thus $x=y$.

(4) implies (1). Since $T \subset \times(x G \mid x \in X), T$ is a compact subset of $X^{X}$. That $T^{2} \subset T$ follows directly from the definition of $T$ and the fact that the maps $t \rightarrow s t(t \in T)$ and $t \rightarrow t g(t \in T)$ of $T$ into $T$ are continuous for all $s \in T$ and $g \in G$. It remains to be shown that given $t \in T$ then it is invertible and that $t^{-1} \in T$.

To this end let $t \in T$. Then $t T$ is a compact subset of $T$ such that $(t T)(t T) \subset t T T \subset \dot{c} T$. Hence by Lemma 1 there exists $u \in t T$ such that $u^{2}=u$. Let $x \in X$ and $\mathscr{F}$ a filter on $G$ such that $\mathscr{F} \rightarrow u$. Let $y=x u$. Then $x \mathscr{F} \rightarrow x u=y$, and $y \mathscr{F} \rightarrow y u=x u^{2}=x u=y$. Hence $x=y$ since $G$ is assumed distal. Thus $x u=x(x \in X)$; that is $u=e$ the identity of $T$.

Since $e \in t T$, there exists $s \in T$ such that $t s=e$. A similar argument applied to $s$ instead of $t$ produces $r \in T$ with $s r=e$. Hence $t=t e=t s r=r$; in other words $t s=s t=e$. The proof is completed.

REMARK. Let $X$ be a Hausdorff space, and let $G$ be a distal group of homeomorphisms of $X$ onto $X$ such that $x G$ is compact for all $x \in X$. Then $G$ is pointwise almost periodic on $X$.

A topological group $G$ is said to be generative provided that $G$ is abelian and is generated by some compact neighborhood of the identity. The remainder of this paper will be concerned with the transformation group $(X, G, \pi)$ where $X$ is a Hausdorff space and the group $G$ is generative.

THEOREM 2. Let $X$ be locally compact zero-dimensional, let $G$ be distal, and let $x G$ be compact for all $x \in X$. Then $G$ is equicontinuous.

Proof. By Theorem 1. $G$ is pointwise almost periodic on $X \times X$. Hence $G$ is locally weakly almost periodic on $X \times X[2 ; 7.07]$, and so $\overline{[(x, y) G} \mid x, y \in X]$ is a star closed decomposition of $X \times X[2 ; 4.16]$. Let $x \in X$ and $\alpha$ an index on $X$. Then $\alpha$ is a neighborhood of $(x, x) G$, and therefore there exists a neighborhood $V$ of $x$ such that $(V \times V) G \subset \alpha$; that is $G$ is equicontinuous at $x$. The proof is completed.

The group $G$ is said to be regularly almost periodic at the point $x \in X$ if given any neighborhood $U$ of $x$ there exists a syndetic subgroup $H$ of $G$ with $x H \subset U$. If $G$ is regularly almost periodic at $x$ for all 
$x \in X$, then $G$ is called pointwise regularly almost periodic on $X$.

THeorem 3. Let $G$ be distal and let $\bar{x} G$ be compact zero-dimensional for all $x \in X$. Then $G$ is pointwise regularly almost periodic on $X$.

Proof. Let $x \in X, U$ a neighberhood of $x$, and consider the action of $G$ on the invariant subset $Y=x G$ of $X$. Let $G_{x}=[g \mid g \in G$ and $x g=x]$. Then $x h g=x g h=x h\left(h \in G, g \in G_{x}\right)$. Hence by continuity $y g=y\left(y \in Y, g \in G_{x}\right)$. For $k \in K=G / G_{x}$ and $y \in Y$ set $y k=y g$ where $k=g G_{x}$. Then $K$ may be regarded as a group of homeomorphisms of $Y$ onto $Y$ such that $x K=Y$. By Theorem 2, $K$ is equicontinuous, therefore $T=$ closure of $K$ in $Y^{Y}$ is a group of homeomorphisms of $Y$ onto $Y$. Hence $T$ is a topological group.

Let $t, s \in T$ such that $x t=x s$. Then since all the maps involved are continuous and $K$ is commutative, $x k t=x t k=x s k=x k s(k \in K)$, hence $y t=y s(y \in Y)$, i.e. $t=s$. Consequently, the map $t \rightarrow x t(t \in T)$ of $T$ onto $Y$ is continuous and one-to-one, hence a homeomorphism. Thus $T$ is compact zero-dimensional.

Now let $V=U \cap Y$. Then $N=[t \mid t \in T$ and $x t \in V]$ is a neighborhood of the identity of $T$. Hence there is an open closed invariant subgroup $L$ of $T$ with $L \subset N$. Since $L$ is open, there exists a finite subset $F$ of $K$ with $T=L F$. Set $M=K n L$. Then $K=M F$ and $M$ is a syndetic subgroup of $K$, such that $x M \subset V$. Consequently $H$, the inverse image of $M$ under the projection of $K$ onto $G / G_{x}$ is the required syndetic subgroup of $G$. The proof if completed.

Theorem 4. Let $X$ be locally compact metric, let $G$ be distal, let $x G$ be compact zero-dimensional for all $x \in X$, and suppose $G$ contains only countably many subgroups. Then the set of points $R$ at which $G$ is equicontinuous is a residual subset of $X$.

As an example of the type of group being considered in Theorem 4 , let $f$ be a homeomorphism of $X$ onto $X$ and set $G=\left[f^{n} \mid n=0, \pm 1, \cdots\right]$.

Proof. Let $\left[H_{n} \mid n=1,2, \cdots\right]$ be the set of syndetic subgroups of $G$, and let $\alpha$ be a metric on $X$. For $m, n$ positive integers set $E(n, m)=$ $\left[x \mid x H_{n} \subset S(x, 1 / m)\right]$ where $S(x, 1 / m)=[y \mid \alpha(x, y) \leqq 1 / m]$, Then $E(n, m)$ is a closed subset of $X$ for all positive integers $n, m$, and $\cup[E(n, m) \mid n=$ $1, \cdots]=X$ by Theorem 3 . Hence $E(m)=\cup[\operatorname{int} E(n, m) \mid n=1, \cdots]$ is an everywhere dense open subset of $X$. Let $E=\cap[E(m) \mid m=1, \cdots]$. Then $E$ is a residual subset of $X$. Moreover, from the definition of $E$, it follows that given any neighborhood $U$ of $x \in E$ there exist a neighborhood $V$ of $x$ and a syndetic subgroup $A$ of $G$ such that $V A \subset U$. Assume $U$ compact and let $K$ be a compact subset of $G$ such that $A K=G$. Then 
$(V \times V) G=(V \times V) A K \subset(U \times U) K \subset(U \times U) G$ shows that $(V \times V) G$ is

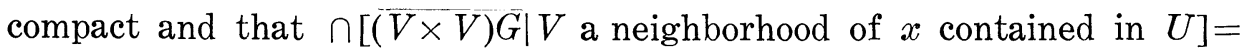
$\cap[(V \times V) G \mid V$ a neighborhood of $x$ contained in $U]$. The proof that $G$ is equicontinuous at $x$ is now completed as in Lemma 1 [1]. Thus $E \subset R$.

The theorems in the second part of the paper suggest the following problems:

(1) Can the assumption that $G$ is generative be dropped in any of these theorems?

(2) To what extent can the condition of zero-dimensionality be relaxed in Theorem 2 ?

The example [1] of a ring of concentric circles rotating at different rates about their common center shows that zero-dimensionality must be replaced by some other condition i.e. cannot be dispensed with entirely even if $X$ is compact. It is conjectured that a sufficient condition would be that $X$ be minimal under $G$; that is that $x G=X$ for all $x \in X$. If this were true then in the general case where all that is assumed is that $x G$ is compact for all $x \in X$, the group $G$ would be distal if and only if $G$ is an equicontinuous family of maps of $x G$ onto $x G$ for all $x \in X$.

The notion of distal was considered by Hilbert see [4] in an attempt to give a topological characterization of the concept of a rigid group of motions. According to the above conjecture and Theorem 1 this would be adequate if $X$ were compact and there existed a point $x \in X$ with $x G=X$.

\section{REFERENCES}

1. W. H. Gottschalk, Characterization of almost periodic transformation groups, Proc. Amer. Math. Soc. 7(1956), 709-712.

2. W. H. Gottschalk and G. A. Hedlund, Topological dynamics, Amer. Math. Soc. Colloquium Publications, 36(1955).

3. A. D. Wallace, The structure of topological semigrous. Bull Amer. Math. Soc. vol 61 No. 21955.

4. L. Zippin, Transformation groups, Lectures in Topology, The University of Michigan Conference of 1940 .

University of Pennsylvania 



\section{PACIFIC JOURNAL OF MATHEMATICS}

\section{EDITORS}

David Gilbarg

Stanford University

Stanford, California

R. A. Beaumont

University of Washington

Seattle 5, Washington

\author{
A. L. Whiteman
}

University of Southern California

Los Angeles 7, California

E. G. Straus

University of California

Los Angeles 24, California

\section{ASSOCIATE EDITORS}

\author{
E. F. BECKENBACH \\ C. E. BURGESS \\ M. HALL \\ E. HEWITT
}

\author{
A. HORN \\ V. GANAPATHY IYER \\ R. D. JAMES \\ M. S. KNEBELMAN
}

L. NACHBIN
I. NIVEN
T. G. OSTROM
H. L. ROYDEN

M. M. SCHIFFER

G. SZEKERES

F. WOLF

K. YOSIDA

\section{SUPPORTING INSTITUTIONS}

\author{
UNIVERSITY OF BRITISH COLUMBIA \\ CALIFORNIA INSTITUTE OF TECHNOLOGY \\ UNIVERSITY OF CALIFORNIA \\ MONTANA STATE UNIVERSITY \\ UNIVERSITY OF NEVADA \\ OREGON STATE COLLEGE \\ UNIVERSITY OF OREGON \\ UNIVERSITY OF SOUTHERN CALIFORNIA
}

\author{
STANFORD UNIVERSITY \\ UNIVERSITY OF UTAH \\ WASHINGTON STATE COLLEGE \\ UNIVERSITY OF WASHINGTON \\ AMERICAN MATHEMATICAL SOCIETY \\ CALIFORNIA RESEARCH CORPORATION \\ HUGHES AIRCRAFT COMPANY \\ THE RAMO-WOOLDRIDGE CORPORATION
}

Mathematical papers intended for publication in the Pacific Journal of Mathematics should be typewritten (double spaced), and the author should keep a complete copy. Manuscripts may be sent to any of the editors. All other communications to the editors should be addressed to the managing editor, E. G. Straus at the University of California, Los Angeles 24, California.

50 reprints per author of each article are furnished free of charge; additional copies may be obtained at cost in multiples of 50 .

The Pacific Journal of Mathematics is published quarterly, in March, June, September, and December. The price per volume (4 numbers) is $\$ 12.00$; single issues, $\$ 3.50$. Back numbers are available. Special price to individual faculty members of supporting institutions and to individual members of the American Mathematical Society: $\$ 4.00$ per volume; single issues, $\$ 1.25$.

Subscriptions, orders for back numbers, and changes of address should be sent to Pacific Journal of Mathematics, 2120 Oxford Street, Berkeley 4, California.

Printed at Kokusai Bunken Insatsusha (International Academic Printing Co., Ltd.), No. 6, 2-chome, Fujimi-cho, Chiyoda-ku, Tokyo, Japan.

\section{PUBLISHED BY PACIFIC JOURNAL OF MATHEMATICS, A NON-PROFIT CORPORATION}

The Supporting Institutions listed above contribute to the cost of publication of this Journal, but they are not owners or publishers and have no responsibility for its content or policies. 


\section{Pacific Journal of Mathematics}

\section{Vol. 8, No. 3 \\ May, 1958}

Michael Israel Aissen, A set function defined for convex plane domaines... . 383

Robert Ellis, Distal transformation groups ................... 401

Ciprian Foias, On a commutative extension of a commutative Banach algebra ....................................... 407

Jerry William Gaddum, Linear inequalities and quadratic forms ......... 411

Allen A. Goldstein and Elliott Ward Cheney, Jr., A finite algorithm for the solution of consistent linear equations and inequalities and for the Tchebycheff approximation of inconsistent linear equations...........

William L. Hart and T. S. Motzkin, Proof of the fundamental theorem on implicit functions by use of composite gradient corrections .......... 429

Henry Berge Helson, Conjugate series and a theorem of Paley .......... 437

Wu-Chung Hsiang, Abelian groups characterized by their independent subsets....................................... 447

John W. Lamperti, On the isometries of certain function-spaces ........ 459

Karel DeLeeuw and Walter Rudin, Extreme points and extremum problems

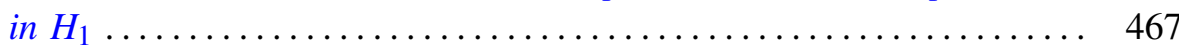

Eugene Lukacs, Some extensions of a theorem of Marcinkiewicz........ 487

George W. Mackey, Multiplicity free representations of finite groups ..... 503

Eben Matlis, Injective modules over Noetherian rings ............. 511

John William Neuberger, Continuous products and nonlinear integral equations

Lawrence Edward Payne and Hans F. Weinberger, New bounds for solutions of second order elliptic partial differential equations...

William T. Reid, A Prüfer transformation for differential systems ........ 575

Howard L. Rolf, The free lattice generated by a set of chains ...

K. M. Saksena, Inversion and representation theorems for a generalized

Laplace integral....................................... 597

Daniel Shanks, Two theorems of Gauss......................... 609

Paul Slepian, On the Lebesgue area of a doubled map ............... 613

Otto Szász and Nelson Paul Yeardley, Jr., The representation of an analytic function by general Laguerre series . ..................... 621

Alan C. Woods, On two-dimensional convex bodies ................. 635 